\title{
Development of an easily interpretable presentation format for meta-analyses in periodontal surgery
}

\author{
D Hauri, PR Schmidlin, * MA Puhan \\ Horten Centre for Patient-oriented Research and Knowledge Transfer and *Clinic for Preventive Dentistry, Periodontology and Cariology, \\ Centre for Dental and Oral Medicine, University of Zurich, University of Zurich, Zurich, Switzerland
}

\begin{abstract}
Problem
The comparison of two therapies is important for dentists so they can support their decisions between available treatment options. Meta-analyses already exist for the comparison of regenerative periodontal therapies. For example, guided tissue regeneration treatments (GTR) using membranes or enamel matrix derivatives have been compared with conservative open-flap debridement without membranes (referred to as standard treatment below). Treatment effects are expressed, for example, as millimetre changes in probing pocket depth (eg, weighted mean difference of $1.21 \mathrm{~mm}$ probing-depth reduction for GTR versus conservative open-flap debridement). ${ }^{1}$ Such treatment effects are rather difficult to interpret for the practitioner because the clinical relevance of these changes is not easily understood. In contrast, eliminating the pockets (to $\leq 3 \mathrm{~mm}$ ) would be considered a clinical success. Therefore, it would be easier to have an estimate of how more likely a clinical success is to be achieved with treatment A than with treatment $\mathrm{B}$.
\end{abstract}

\section{Objective}

Our aim was to develop a presentation format for meta-analyses that is easy to interpret for dentists in situations where there is an agreed-upon definition of clinical success.

\section{Methods}

We re-analysed two meta-analyses that included randomised trials, reported in $\mathrm{Tu}$ et al. ${ }^{2}$ One of them compared GTR (15 trials) with open-flap debridement without additional membranes and the other one trialled the use of enamel matrix derivatives (10 trials) against open-flap debridement without additional membranes. From each study, we extracted the number of participants and mean pocket depths with their standard deviation at followup. We had to exclude some of these studies (five GTR and one enamel matrix derivative trial) for our meta-analysis because they only calculated and reported differences between baseline values and followup values, but did not report on mean changes and standard deviations that were necessary for our analysis. We then calculated the number of patients with pocket depths $\leq$ or $>3 \mathrm{~mm}$ as follows. We assumed that the pocket depths at followup were distributed normally. Based on the mean pocket depths and their standard deviation, we calculated probabilities for clinical success, that is, for pockets $\leq 3 \mathrm{~mm}$ (formula explained in Appendix).

Based on these probabilities, the number of people with and without clinical success ( $\leq$ or $>3 \mathrm{~mm}$ ) were calculated by multiplying the number of participants by the calculated probabilities. Using the resulting proportion of patients with and without clinical success we used random-effects meta-analysis to estimate pooled odds ratios (OR) for clinical success (figures 1 and 2).

\section{Results}

The mean probability for clinical success was $43 \%$ for GTR, $26 \%$ for the use of enamel matrix derivative and 13\% for standard treatment. GTR was highly effective at reducing pockets compared with standard treatment [pooled OR, 6.4; 95\% confidence interval (CI), 2.9-14.0; I 2 51\% (suggesting moderate to large between-study heterogeneity)]. Enamel matrix derivative was only slightly more effective than the standard treatment (pooled OR, 1.6; 95\% CI, 1.0-2.5; $\mathrm{I}^{2} 0 \%$, suggesting no betweenstudy heterogeneity).

\section{Conclusion}

The meta-analyses showed that GTR increased the chance of clinical success about sixfold compared with standard treatment, whereas enamel matrix derivatives were only slightly better than standard treatment. This alternative of analysing and presenting randomised trials offers a much easier interpretation than a presentation

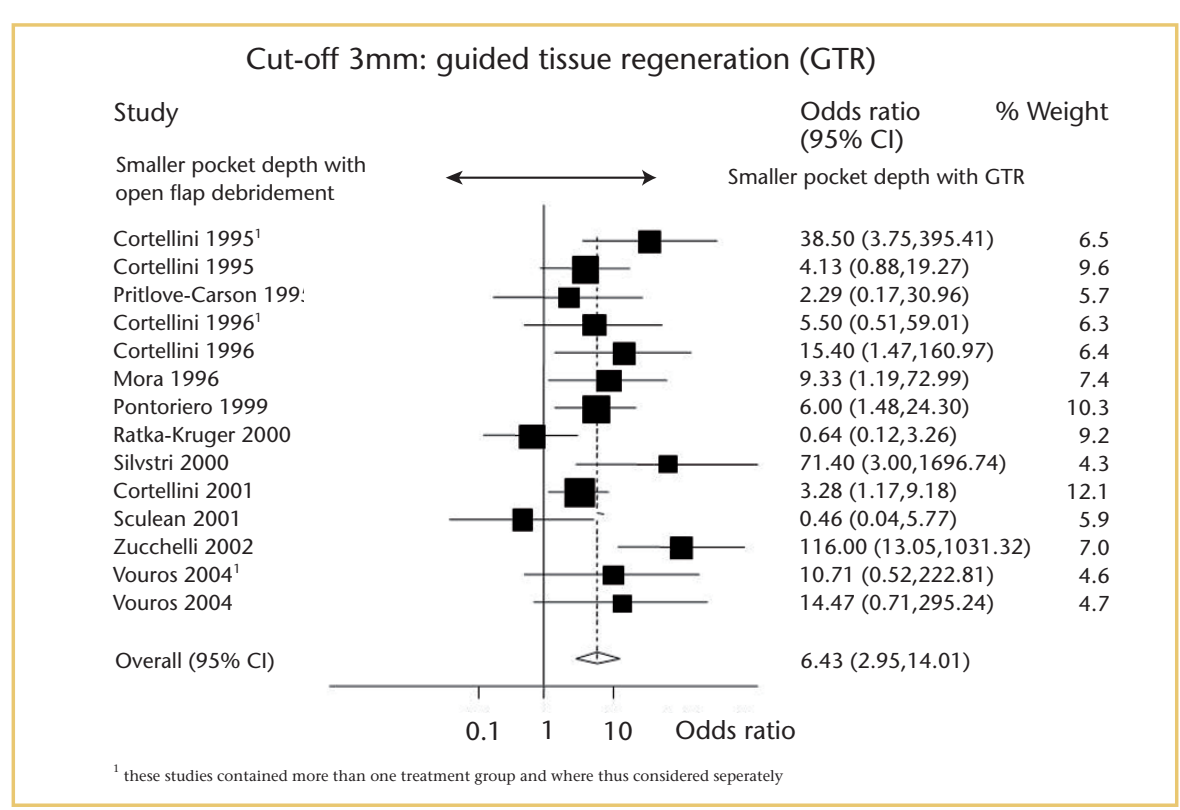

Figure 1. Forest Plot for guided tissue regeneration (GTR) studies 
of weighted mean differences in millimetre changes. In addition, the calculation of probabilities, based on the assumption of normality, is simple.

A weakness of our approach is that the calculated probabilities are based on the assumption that the data are distributed normally. This cannot be verified without having access to the original data. If data are not distributed normally some misclassification will occur, but randomisation might minimise this problem because the extent of misclassification is likely to be similar in the treatment groups. In general, it is more adequate to keep continuous data and not to dichotomise them because continuous data carry more information. In situations where interpretation of continuous outcomes are difficult, however, and where clear cutoffs exist determining clinical success, a transformation as described above can facilitate interpretation substantially. Of course, the best analysis would be based on randomised trials that themselves reported the number of participants achieving (or not) clinical success. Since it is unrealistic to expect all studies to report clinical success rates, however, our approach proposes a solution by presenting results of meta-analyses in a more easily understandable format.

1. Needleman IG, Worthington HV, Giedrys-Leeper E, Tucker RJ. Guided tissue regeneration for periodontal infra-bony defects. Cochrane Database Syst Rev 2006; issue 1.

2. Tu Y-K, Tugnait A, Clerehugh V. Is there a temporal trend in the reported treatment efficacy of periodontal regeneration? A meta-analysis of randomised controlled trials. J Clin Periodontol 2008; 35:139-146.

3. Stahel WA. Statistische Datenanalyse. Eine Einführung für Naturwissenschaftler. Wiesbaden: Vieweg Verlag; 2002.

Evidence-Based Dentistry (2008) 9, 89-90 doi:10.1038/sj.ebd.640604

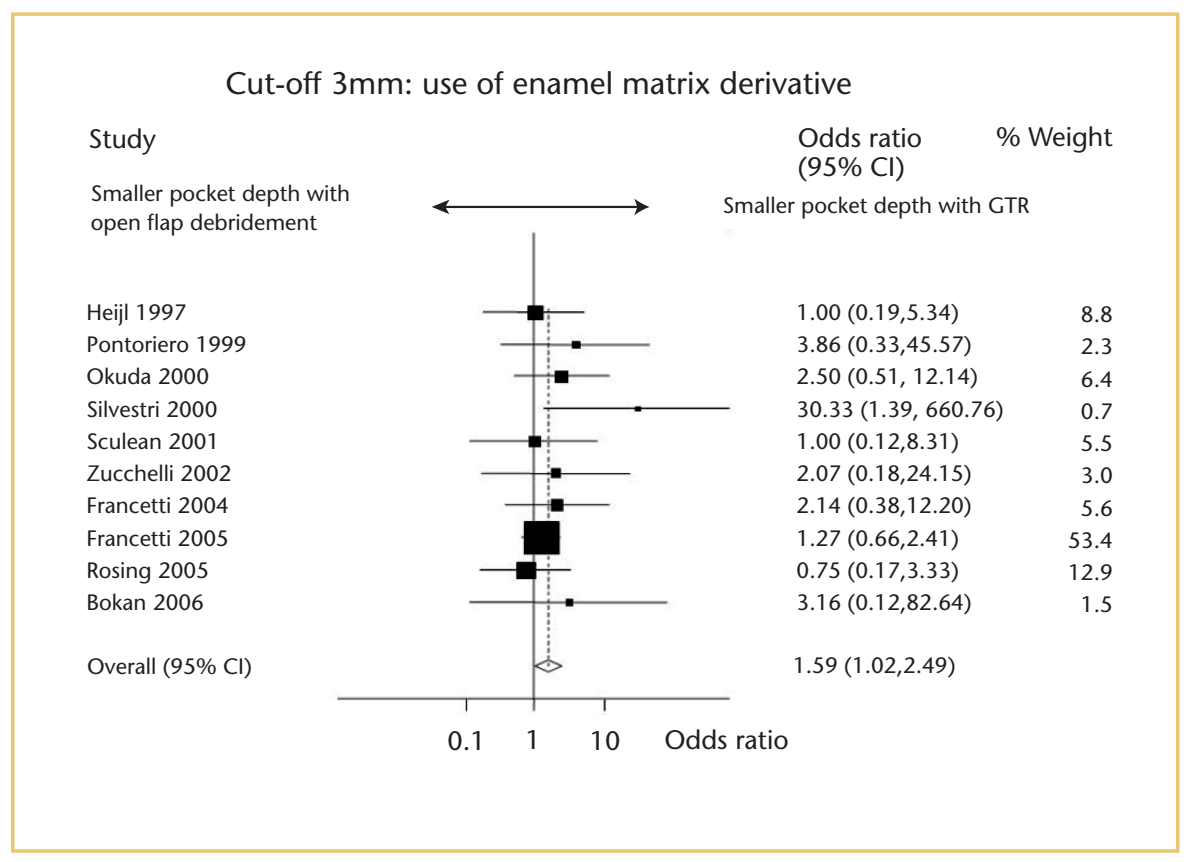

\section{Figure 2 . Forest Plot for enamel matrix derivative studies}

\section{Appendix}

The formula for the calculation of the probabilities $\mathrm{P}(\mathrm{X} \leq \mathrm{x})$ for clinical success, ie, for pockets $\leq 3 \mathrm{~mm}$ is as follows:

$$
\mathrm{P}(\mathrm{X} \leq \mathrm{X})=\mathrm{F}(\mathrm{X})(\mathrm{x})=\phi((\mathrm{X}-\mu) / \sigma)
$$

with $\mathrm{F}(\mathrm{X})(\mathrm{x})$ as the cumulative distribution function, $\mu$ as the mean, $\sigma$ as the standard deviation and $\mathrm{x}$ as the cutoff value, eg $3 \mathrm{~mm}$.

For the expression:

$$
\phi((\mathrm{x}-\mu) / \sigma)
$$

one can ascertain the probabilities by consulting normal distribution tables as described in Stahel. ${ }^{3} \phi$ stands for the distribution function of the standard normal distribution. The probability values in the Tables published by Stahel ${ }^{3}$ are only valid for standard normal distribution. For example, for a group of nine patients, the mean pocket depth at followup is $3.53 \mathrm{~mm}$ with a standard deviation of 0.62 . Therefore, for:

$$
\phi((\mathrm{x}-\mu) / \sigma)
$$

we get $((3.00-3.53) / 0.62)=-0.85$. The probability for pockets $\leq 3 \mathrm{~mm}$ is 0.20 , as derived from the Table in Stahel. ${ }^{3}$ Hence, the number of patients with pockets $\leq 3 \mathrm{~mm}$ is $9 \times 0.20$ is approx. two and the number of patients with pockets $>3 \mathrm{~mm}$ is $0.80 \mathrm{x} 9$ is approx. seven. 